\title{
An Off-the-Shelf Platform for Automatic and Interactive Text Messaging Using Short Message Service
}

\author{
Daniel Oliveira $^{1}$, Diana Oliveira ${ }^{3}$, Nuno M. Garcia ${ }^{1,2,4}$, and Graça Esgalhado ${ }^{3}$ \\ ${ }^{1}$ Computer Science Department, University of Beira Interior, Covilhã, Portugal \\ ${ }^{2}$ Instituto de Telecomunicações, Covilhã, Portugal \\ ${ }^{3}$ Psychology and Education Department \\ Universidade da Beira Interior, Covilhã, Portugal \\ ${ }^{4}$ Universidade Lusófona de Humanidades e Tecnologias, Lisbon, Portugal \\ danieloliveira@it.ubi.pt, dianapsoliveira@gmail.com, \\ ngarcia@di.ubi.pt, mgpe@ubi.pt
}

\begin{abstract}
This paper describes the design and construction of a platform for the implementation of an automatic and interactive message handling system through the use of Short Message Service (SMS), also known as texting, devised to be used as support of a Psychology study. The platform was devised to use low cost off-the-shelf parts, yet allowing the design of an efficient and robust system. The research that prompted the platform's construction included researchers from the Psychology and Education Department and the Computer Science Department of the University of Beira Interior. The study's goal is to assess psychological changes of the research subjects after exposure to motivational SMS texts. The paper describes the strategies adopted to design the architecture of the platform and the setting in place of the system, including the description of the used software and hardware. The source code of this system is publicly available at the Assisted Living Computing and Telecommunications (ALLab) website.
\end{abstract}

Keywords: SMS, messaging, mobile, off-the-shelf, psychology, self-regulation, self-efficacy.

\section{$1 \quad$ Introduction}

It is considered that information and communication technologies play a major role in all aspects of current human interactions in the so-called developed countries and in particular for young humans.

Nowadays, mobile devices seem to be the first choice of graduate students to communicate, organize, actualize and eventually learn [1], in particular if smart phones are considered. When taken into account that only a very small percentage of students are not avid texters, we can assume that text messaging is one of the most privileged ways they use to communicate [2]. That might be why, even though the use 
of this technology is still taken with caution by colleges and lecturers, in the past decade there have been several studies concerning the use of Short Message Service (SMS) in an educational context. So far SMS technology has been used to send students administrative information [3], some educational content [4,5], or to send persuasive and motivational quotes [6], just to name some of its applications. Yet, there's not much research in this particular area, therefore we are just starting to analyze how SMS may be used as a tool to support teaching and learning in higher education.

With that in mind, in the context of research in the areas of Computer Science (CS) and Pedagogical Supervision (PS) at the University of Beira Interior (UBI) it was devised a joint research to study whether persuasive and motivational SMS texts could be used to increase the student's performance in mathematics classes, of students from four different undergraduate courses. This was done through a study that focused in establishing the effects of the SMS intervention in the student's selfregulated learning, general academic self-efficacy and learning strategies, and involved the definition of the architecture supporting that study.

This paper focuses mainly in the description of the computational issues of the study, although some other topics will be introduced to contextualize. As the results of the psychology study are not available at this time, it's not the goal of this paper to present conclusions regarding the impact of the use of the platform in the study subjects. The remainder of the paper is organized as follows: this paragraph concludes section 1; Section 2 presents a very brief overview of the state of the art; Section 3 describes the system's architecture; Implementation is discussed in Section 4; Section 5 concludes the paper, and references and acronyms are shown in the final part.

\section{State of the Art}

Globally, in the last decade, the telecommunications field has experienced a great increase not only in network coverage, but also in technological innovation [7]. Furthermore text messaging is now so popular, it is being used by people of all ages and technology expertise, and among university students texting is generalized $[2,4]$.

Importing something as widespread as SMS and adapting it as a tool for teaching and learning purposes is only natural [2] considering we're not introducing something entirely new to colleges and classrooms.

Today's students use mobile phones and SMS, inside and outside school, therefore, the research was developed to assess whether SMS could have a motivational impact, as suggested by previous research [4,8].

In recent years, colleges and other higher education institutions have tried to come up with ways to introduce mobile phones in ways that may enhance lecturers supported in pedagogy. This means that from a pedagogical perspective, there may be opportunity to raise student engagement, considering the subjects studied at a higher education level [7] and use pervasive technologies such as the SMS.

The popularity of SMS is believed to have inspired some lecturers to explore their use in the educational context [9]. Besides studies that connect the usage of SMS with weight loss [10,11] and smoking cessation [12], in recent years investigators from the educational field have turned their sights into how this technology can be used in a producible way in their own work places: high education institutions. We can find 
such studies in many parts of the world, but as not to be exhausting some examples of such usage can be found in the following research: [3] published a study on how SMS could be used to send administrative content to students; [4,5] that focused on how to send educational contents through SMS; authors in [13] focused not only in the delivery of educational content, but also on how that might affect student engagement to class assignments; and [14] focused on how SMS exchange in class could increase its interactivity.

The development of the platform used in this study was made taking into consideration the requirements defined by the main stakeholders, the Pedagogical Supervision Master of Science (MSc) student and their supervisors. Although we found some commercial solutions that fulfilled some of the requirements, we didn't find an integrated solution that complied with them all and since we had a tight budget and tight time schedule to start the study, creating our own system architecture from the ground up was the obvious choice, especially when taking into consideration that there was previous expertise in this area and several in-house projects had been already developed at an experimental level.

\section{System Architecture}

The system was built taking into consideration the requirements and challenges posed by the psychology study it would be used for: (1) the system needs to work uninterrupted and unattended; (2) the system needs to address Mobile Network Operator (MNO) fair use policies, by not sending large amounts of SMS texts in a very short period of time; (3) the students have to receive a stream of messages at predefined times; (4) some messages require answers making this an interactive questionresponse system.

To comply with the requirements, a mechanism to control the message flow for each student was needed, i.e., as each student responds on its own time, the message being processed for a particular student may be different from the message of the student that precedes him/her in the message queue.

The stream of SMS messages as well as the list of destinations were inserted as elements of a database (DB). The questions themselves, the admissible answers and the message flow was defined based on psychological validated literature as expressed in studies aiming to evaluate self-regulated learning and/or self-efficacy in the same population[17,18], which will be explored outside this paper.

To manage this flow of messages, a script was created which connected questions with their validated answers, and when admissible, gave predefined replies according to admissible options presented to the study subjects. After this process, discussed in more detail in the architecture section the script advances to the following question.

As to hardware it was decided to use three Global System for Mobile Communications (GSM) modems, one for each major Portuguese MNO: MEO (formerly known as TMN), Vodafone and NOS (formerly known as Optimus) (all trademarks and brands are property of their respective owners). This choice of operators was made because the researchers were confident that almost all students had subscribed to one of the free SMS plans that all MNO offer, which guaranteed that the research would be carried out with no costs to the end user, even when answers were required. 
The modems were connected to a laptop via a powered universal serial bus (USB) hub, because the modems were too large to connect directly to the laptop and because that allowed extra power needed to support the modems, since the hub has an external power supply. To assure that the laptop was always ready to exchange SMS, an Uninterruptible Power Supply (UPS) was added to the hardware to increase power supply resiliency. The setting and operation of the solution cost $625 €$, as follows: Capital Expenditure (CAPEX) summed $505 €$ by adding $300 €$ for the small laptop used, $100 €$ for the UPS, $5 €$ for the USB hub, and $75 €$ for the three GSM modems ( $25 €$ each in average); Operational Expenditure (OPEX) summed $120 €$ (30€ per month), to allow the payment of $10 €$ per each MNO subscription (these values refer to early 2014 and include all legal taxes but not electricity expenses). Each Subscriber Identity Module (SIM) card was purchased with a free SMS plan, so sending messages had no cost. The architecture of the system is shown in Fig. 1.

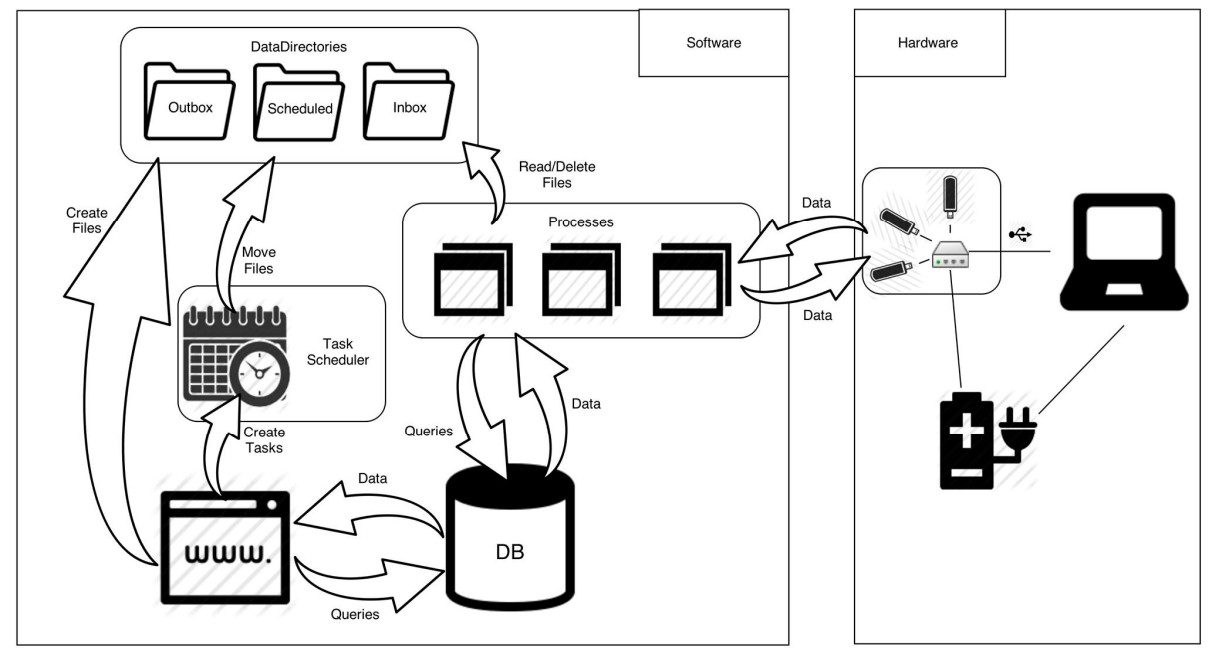

Fig. 1. System architecture diagram

Concerning software, a decision was made to assign the SMS exchange functionalities and the handling of the received SMS's to two separated processes, both sharing access to the three directories created for each MNO (Fig. 2). Because each process only has access to the directories of the assigned operator, each user only receives SMS sent from the operator. As depicted in Fig. 1, the computer runs six processes, each interacting with the DB, the computer's file system and one of the MNO GSM modems. One group of processes is in charge of processing the received messages and replies and implementing the interactive feature of the system. The other group of processes is in charge of sweeping its associated GSM modem, looking for incoming messages and retrieving and storing these messages in the respective Inbox folder. This group of processes is also in charge of sweeping the Outbox folder and sending the found outgoing messages to its associated GSM modem. More details on the tasks performed is given in Section 4. 
The system also relies on (Fig.1):

- a web application to schedule the SMS's, add/remove users and disable the dispatch of messages to a given user, among others functionalities;

- a DB to store the messages (text) to be scheduled, the auto-reply messages, the users' information, the replies received from the users and all additional support tables;

- a task scheduler that creates the tasks requested from the web application.

No comparison between this solution and others available is given because most of them aren't neither free not open-source, which prevents comparability concerning, the underlying used architecture.
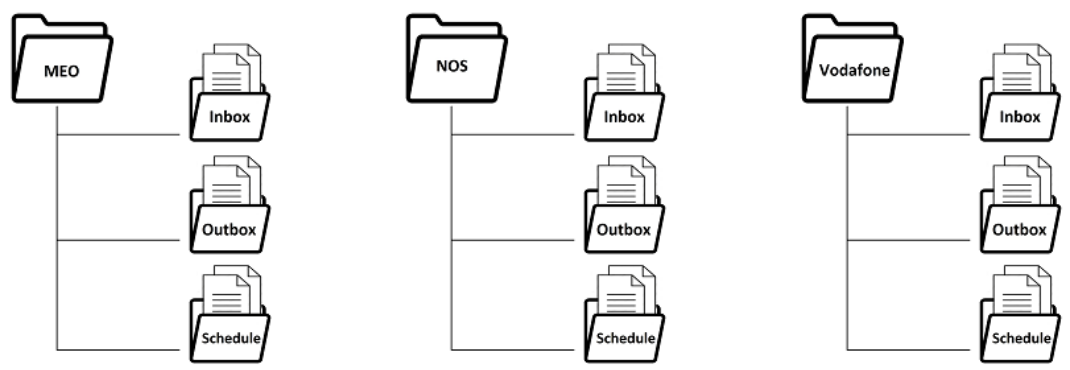

Fig. 2. Directories tree for each Mobile Network Operator

\section{$4 \quad$ Implementation}

The research started when MSc students from the DPE decided to develop their dissertations on the impacts of new technologies in the educational environment, but only the study entitled "The efficacy of the intervention through SMS in the learning's self-regulation and general academic self-efficacy in ungraduated students" will be mentioned.

The study focus on the use of SMS with persuasive and supportive messages to students from different courses: Computer Science Engineering, Electromechanical Engineering, Electrical and Computers Engineering and Optometry - Vision Sciences, which have in common poor academic performance and high dropout rate on their respective mathematics class. A three stages process was followed: a pre-study, the experiment, and a post-study. Both in the pre and post studies, the students were invited to fill a socio-demographic and data questionnaire prepared and managed by the DPE researchers and the experiment itself involves the use of the tool targeted by this paper. This study also included other relevant questions such as the students' mobile phone number and the desired MNO it would communicate with.

The pre-study started by initially contacting teachers who lectured math to the research subjects. The teachers were asked to collaborate and to make available 20 minutes of their first class of the semester for the researchers to invite students to participate in their study. Afterwards, the complete questionnaires were separated by course and class for the purpose of creating an experimental and a control group, 
where all classes and courses were represented: students were randomly rafted to one of the groups, by course and class.

Around 300 students were invited to participate, but only 184 filled the initial prestudy questionnaire; this roughly corresponds to the number of students met when visiting the classes at the start of the semester.

Considering that along the semester dropouts might occur, we didn't sort the students equally between our experimental and control group, instead we sorted 110 students $(60 \%)$ to the experimental group and the other $74(40 \%)$ to the control group.

During the experiment stage, while students normally attended classes, students rafted to the experimental group received a SMS twice a week, on Mondays and Thursdays and those of the control group didn't.

At the end of the semester the teachers lecturing the mathematics class were again contacted and the students were invited to fill the same questionnaire, by course and by class.

Regarding the computational side of the work, there were several decisions that led to the system architecture being discussed. All the software was developed using C\# since it enables developers to easily create applications that run on the .NET Framework and there is previous experience with this language and framework.

At a later time, outside the scope and goal of this paper, researchers will compare scores between what students responded on the questionnaires pre and post-study, aiming to analyze differences between the experimental and control groups, in an attempt to assess whether receiving the texts modified the way students answered to the same set of questions.

\subsection{Processes}

The choice to create two different processes for each MNO resulted from the analysis made to the time spent between exchanging SMS messages, handling of received messages, including creating an answer if needed, saving the message, etc., since it wasn't desirable that the solution stopped exchanging SMS texts each time it had to handle received texts, whether they came from students, or operators (promotions, balance alerts, etc.). It was decided to call each of the processes "SMS Gateway" (send/receive) and "Auto-reply agent" (manage received messages). Along with time management, the other rationale for the separation of these two processes was to allow a layer of isolation between the management of the messages on the GSM modems (inbound and outbound), the management of messages received from a user, and the implementation of the necessary interactivity feature.

The "SMS Gateway" searches for new files in the "Outbox" directory of the assigned MNO. If it finds a new file it will process it by sending the SMS text to the recipients contained in the file, processing files in a last in first out manner using for this the time stamp for the creation of the file.

Since the modem cannot send and receive messages at the same time, it was decided to insert a delay between the dispatch of each message, not only to keep the buffers from overflowing and resulting in a faulty service, but also to allow incoming messages buffered at the MNO system to be delivered to the GSM modem and retrieved and stored in the respective folder ("Inbox" folder). To further take advantage 
of the delay, this time lapse is used to verify if any other message has been received at the modem, and if so download it from the SIM card to a file in the Inbox directory created for this purpose, (c.f. Fig. 2). The SMSLib [15] library was implemented in the gateways to easily exchange messages through the GSM modems, since it is an Application Programming Interface (API) available and tested in the Microsoft .Net framework.

The "Auto-reply agent" handles the received messages by always watching the "Inbox" directory for new files. If it finds one or more files it will decide how to handle them according to the system specifications: store it, delete it or answer it.

Internally, each of the outgoing message files has the following structure: FLOW_ID*MESSAGE*LIST_OF_RECIPIENT_NUMBERS. In the given example "230" represents the flow id of that message, the text to be sent spans from "SMS30UBI:" until "participaste." and +351000000000, +351111111111 and so on are the recipients' phone numbers, as shown in Fig. 3. The asterisk character "*" was used as field delimiter.

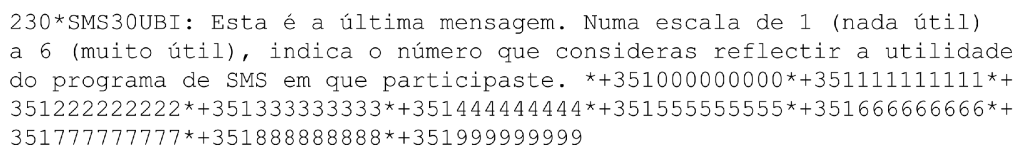

Fig. 3. Outgoing message example (in Portuguese, phone numbers anonymized)

The list of text recipients is never larger than 10, in order to allow the GSM modems to receive incoming messages as the FAQ available in the SMSLib webpage suggests that if the library is being used with GSM modems the rate at which it can send SMS is about 6 messages per minute (a message each 10 seconds). However a compromise was reached to send each message with a random interval of 10 to 15 seconds in order to respect the fair use policy that each MNO demands from its' users. By limiting the amount of messages the system can send at once, it is guaranteed that no high priority message has a delay of more than 150 seconds before being sent. This is particularly relevant as the study aimed to implement a responsive interactive system to the user's point of view, thus allowing that, in the worst case scenario if a user's response is inversely buffered and all users respond at the same time to the received message, no user is left without response for more than 40 seconds.

As each individual message is part of a predefined flow, and each user may interact with the system at different points of each flow, the different messages have to be identified by a flow identifier (flow id). The flow id identifies the message that is being sent, giving also information as to if this message is waiting for an answer and what is the auto-reply to be delivered if the received answer is valid. The degree of interactivity of the system is limited to one question, several possible answers, and several corresponding replies, i.e., the dialog between the user and the system is limited to the depth of question-response-acknowledgment messages (Fig. 4). Nevertheless, more complex flows can be devised and stored at the DB without any influence to the complexity of the other parts of the system itself.

Inside a message, the numbers in the list of recipients are separated by the same control character used to separate the other fields (* in this example). The real 
control character is an extended ASCII character, chosen because it is highly improbable that it would be used in the text of a message, preventing a risk/abuse of the system. Message text is encoded in UCS2, a Unicode encoding that uses a 16-bit character format and allows accented characters along with other special ones (like c with cedilla 'ç'), in spite of losing the maximum length for each message (from 160 to 70 characters).
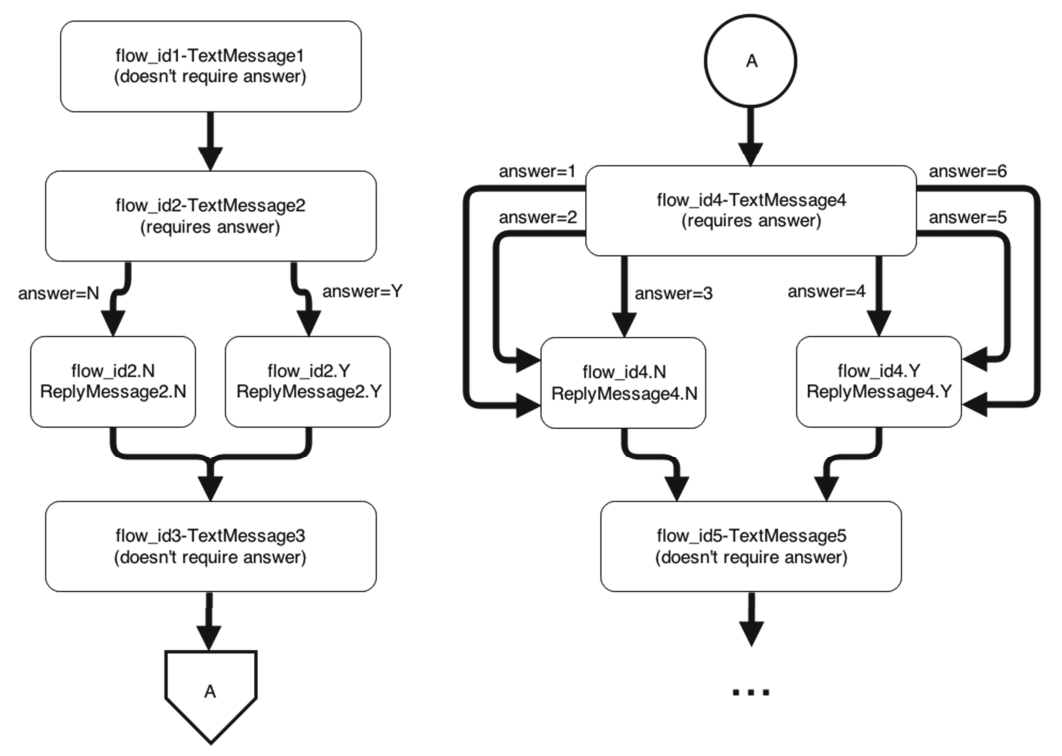

Fig. 4. Abstract example of message flow

A scheme for the processing of messages is presented in Fig.5 and consists of the system sending a message, leaving the system waiting for an answer. Assuming that the message is received by the recipient, two scenarios might occur: (1) the recipient sends one of the required response answers, this message is processed and a new message (the corresponding auto-response) is issued; (2) the recipient responds when no reply was expected: the response message is stored at the DB for further inspection and an alarm is raised in the control panel of the system, allowing the unexpected reply to be viewed by the system's administrator. If a response is expected but the user does not respond in a period of 1440 minutes ( 24 hours), the system administrator can decide what to do, for example, advance the flow of the user or send the message again (Fig.5).

\subsection{Web Application}

The application's main objective is to manage the scheduling of outgoing messages using a task scheduler, or preparing a message to be sent immediately, by creating files which contain the information needed to send a SMS. Since in this study the same message might be sent to several students at the same time, it was decided to 
create batches of files and each file has at most ten recipients. This way the process processes a file with many recipients and does not consider an interruption to allow, for example, the dispatch of a message might have a higher priority. Yet, as there are several processes running in parallel, there may be the case where all three modems are sending messages at the same time.

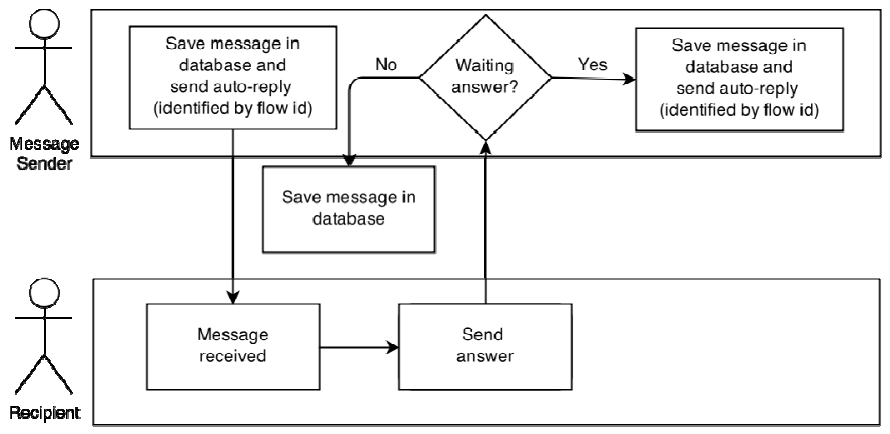

Fig. 5. Message flow scheme

The referred files are created in the respective MNO "Outbox" folder if it's a message to be sent immediately or in the "Scheduled" folder if it's a message destined to be sent later, usually, at a predefined time. When a scheduled message is created, the web application also creates the respective task in the Scheduler, including the details concerning the file's original location, when to move it, and to what directory it should be moved. In this study it was decided that the initial messages should always be sent between $18: 30 \mathrm{~h}$ and $20: 30 \mathrm{~h}$, as not to disturb classes or sleep of the recipients.

\subsection{Database}

Microsoft SQL server was chosen since it has an easy integration with the web application (uses the same platform, the .NET framework), and it was used to save users information (message recipients), messages flow, log the messages exchanged with each user, courses' information, among other aspects, and its structure is shown in Fig. 6.

The following tables are responsible for the message flow control:

- Fluxo_EI - Has information about the flow identifier of each message, along with the respective message, response for a positive answer, for a negative answer and the type of message (if the message requires an answer or not);

- Fluxo_saida - Saves the sent message for each recipient, together with the respective timestamp, the flow at which corresponds and if the recipient has replied to the message;

- Fluxo_entrada - Used to store received messages for each recipient, along with the respective timestamp, the flow at which it corresponds and the text received. 


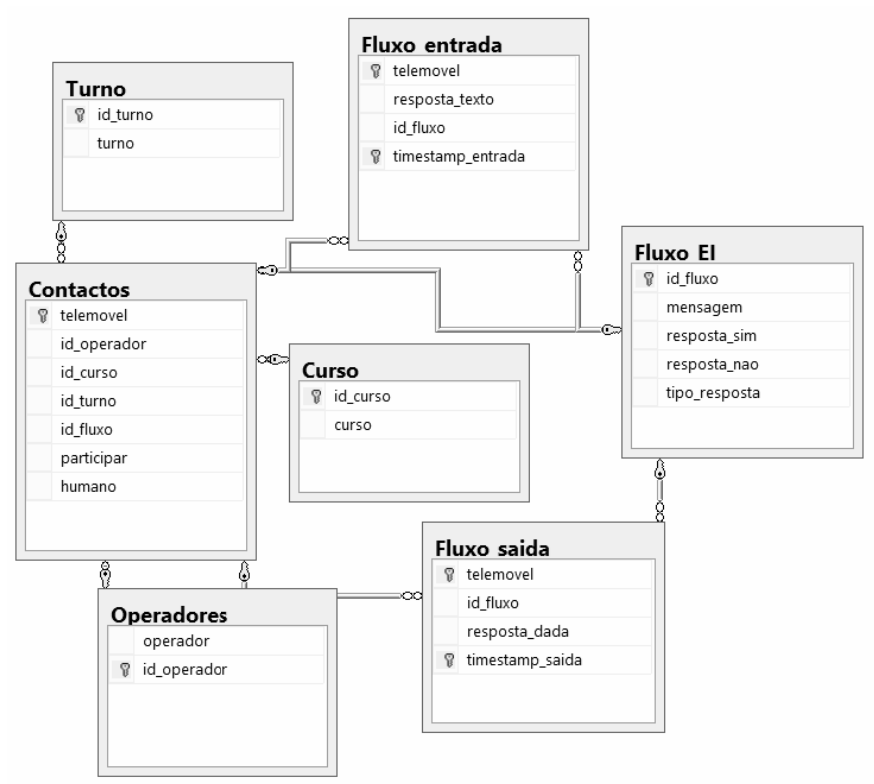

Fig. 6. Database table structure

\subsection{Task Scheduler}

This component creates tasks via the web application consisting of the following: for a given task, when the scheduled time arrives, the task scheduler will move the file defined in the task from the "Scheduled" directory to the respective "Outbox" directory, which will then be found by the process that handles the outgoing messages ("SMS Gateway"), sending them to the respective recipients.

It was decided to use the task scheduler available in the laptop operating system (Windows 7) on the grounds that this was more than adequate for the needed feature, therefore avoiding the creation of a proprietary task scheduler. This decision was also possible because the computer used for this task was dedicated to this study alone and no other scheduled tasks were added by other software components.

\section{$5 \quad$ Conclusion and Future Work}

Although the final results of the psychological study are neither the focus of this paper, nor available at this time, we may draw conclusions as to functionality of the support platform.

The auto-reply response time was generally perceived as very quick by the developers and the users, especially considering that it was an academic solution using low cost off-the-shelf hardware and simple software. Yet, one student complained that he couldn't read the texts. However when further questioned he told the researchers he was using a very old mobile phone that he no longer had with him, making it impossible to identify a possible solution to this problem. In future tests, the researchers will 
devise a pre-test to confirm that every subject is able to receive messages in a readable format.

Taking in consideration that we have sent and received a considerable number of messages (almost 7000), we conclude that the system ran as expected, having an uptime of $100 \%$ and zero system crashes. All this was achieved using a low-end laptop (Intel Atom N450, 1.66GHz clock, 2GB of RAM and a 64-bit operating system), having a workload of almost $100 \%$. Moreover, the design of the messages and the request for replies was praised by several test subjects for being simple, user-friendly and not being too time consuming. The developed software source code is available at our research laboratory's MediaWiki [16]. The fact that the system was built using old off-the-shelf parts and components, and even so allowed for a free communication between the system and the users, makes it clear that the system was the exact response the requirements set. The system aimed not to abuse the MNO network with massive SMS messages and the fair use principle that applies to each of the subscribed plans was not violated.

It should be pointed out that the simplicity of this system allows for it to be easily adapted to other studies, subjects or businesses, not being limited to students or even academic environments (with the respective login account creation and credentials).

Acknowledgments. The authors would like to acknowledge the contribution of the COST Action IC1303 - AAPELE. This work was supported by FCT project PEstOE/EEI/LA0008/2013.

\section{References}

1. Mellow, P.: The media generation: Maximize learning by getting mobile. ascilite 2005: Balance, Fidelity, Mobility: maintaining the momentum? p. 469-475 (2005), http: / /www .ascilite.org.au/conferences/brisbane05/blogs / proceedings /53_Mellow.pdf (retrieved March 12, 2014)

2. Lominé, L.L., Buckhingham, C.: M-learning: texting (SMS) as a teaching \& learning tool in higher arts education. ELIA Teachers' Academy, Sofia. p. 1-6(2009), http://www.elia-artschools.org/images/activiteiten/18/files/ Lomine $\% 20-\% 20$ Texting $\% 20$ as 20 a 20 tool\%20 for 20 teaching . pdf (retrieved March 12, 2014)

3. Naismith, L.: Using text messaging to support administrative communication in higher education. Active Learning in Higher Education 8(2), 155-171 (2007),

http: //ganymedes. lib.unideb.hu:8080/udpeer/bitstream/2437.2/ $11817 / 1 /$ PEER_stage2_10.1177\%252F1469787407078000.pdf (retrieved March 12, 2014)

4. Lu, M.: Effectiveness of vocabulary learning via mobile phone. Journal of Computer Assisted Learning 24, 515-525 (2008)

5. Zhang, H., Song, W., Burston, J.: Reexamining the effectiveness of vocabulary learning via mobile phones. TOJET: The Turkish Online Journal of Educational Technology 10(3), 203-214 (2011), http://www.tojet. net/articles/v10i3/10323.pdf (retrieved March 12, 2014) 
6. Goh, T., Seet, B., Rawhiti, L.: Persuasive and Affective SMS text messaging for Students' Learning (2011), http://akoaotearoa.ac.nz/download/ng/file/group$6 /$ persuasive-and-affective-sms-text-messaging-for-studentslearning.pdf (retrieved October 16, 2013)

7. Mockus, L., Dawson, H., Edel-Malizia, S., Shaffer, D., An, J.S., Swaggerty, A. (2011), The Impact of Mobile Access on Motivation: Distance Education Student Perceptions. Learning Design at Penn State's World Campus. World Campus Learning Design. p. 1-34, http: / / learningdesign.psu.edu/research/MLRTWhitePaper.pdf (retrieved March 12, 2014)

8. Thüs, H., Chatti, M.A., Yalcin, E., Pallasch, C., Kyryliuk, B., Mageramov, T., Schroeder, U.: Mobile Learning in Context. International Journal of Technology Enhanced Learning 4(5/6), 332-344 (2012), http: / / learntech.rwth-aachen. de/d11154 (retrieved November 20, 2013)

9. Gasaymeh, A.M., Aldalalah, O.M.: The Impact of Using SMS as Learning Support Tool on Students' Learning. International Education Studies 6(10), 112-123 (2013)

10. Brug, J., Oenema, A., Kroeze, W., Raat, H.: The internet and nutrition education: challenges and opportunities. European Journal of Clinical Nutrition (59), 130-139 (2005)

11. Wang, Y., Tim, L.: Worldwide trends in childhood overweight and obesity. International Journal of Pediatric Obesity 1(1), 11-25 (2006)

12. Free, C., Knight, R., Robertson, S., Whittaker, R., Edwards, P., Zhou, W., Rodgers, A., Cairns, J., Kenward, M., Roberts, I.: Smoking cessation support delivered via mobile phone text messaging (txt2stop): a single-blind, randomised trial. The Lancet 378(9785), 49-55 (2011)

13. West, D.M.: Mobile Learning: Transforming Education, Engaging Students, and Improving Outcomes. Center for Technology Innovation at Brookings. Mobile Learning, 1-17 (2013)

14. Markett, C., Sánchez, I.A., Weber, S., Tangney, B.: PLS Turn UR Mobile On.: Short message service (SMS) supporting interactivity in the classroom pp. 1-5 (2004)

15. SMSLib - A universal API for sms messaging, http://smslib.org (last accessed June 05, 2014)

16. Oliveira, D., Garcia, N.M.: Interactive SMS Agent http: / / allab.it.ubi.pt/mediawiki (last accessed June 10, 2014)

17. Biggs, J.B.: The Study Process Questionnaire (SPQ): Manual. Hawthorn, Vic.: Australian Council for Educational Research (1987)

18. Torre Puente, J.C.: La autoeficacia, la autorregulación y los enfoques de aprendizaje en estudiantes universitarios. Tesis doctoral, Madrid, Spain: Universidad Pontificia Comillas (2006) 